\title{
PERFIL DEL PACIENTE HIPERFRECUENTADOR Y SU ASOCIACIÓN CON EL TRASTORNO ANSIOSO DEPRESIVO EN SERVICIOS DE ATENCIÓN PRIMARIA DE CALI, COLOMBIA
}

\author{
Mérida Rodriguez-Lopez ${ }^{1,2, a}$, Marcela Arrivillaga ${ }^{3, b}$, Jorge Holguín ${ }^{3, ~ c}$, Hoover León ${ }^{4, ~ d, ~ A l f o n s o ~ A ́ v i l a ~}{ }^{5, \text { e }}$, \\ Carlos Hernández ${ }^{2,6, f}$, Hernán G. Rincón-Hoyos ${ }^{7,8, g}$
}

\begin{abstract}
RESUMEN
Objetivos. Determinar el perfil de los pacientes hiperfrecuentadores en servicios de atención primaria y su asociación con el trastorno ansioso depresivo en Cali, Colombia. Materiales y métodos. Estudio de casos y controles. Se definió como caso hiperfrecuentador a aquellos pacientes con un percentil $>75$ en la frecuencia de uso de consulta externa por demanda espontánea en los últimos 12 meses y como controles a aquellos con percentil <25. Se utilizó el análisis de correspondencias múltiples para describir los perfiles de los pacientes y mediante regresión logística se determinó la influencia de la depresión y ansiedad en la hiperfrecuentación. Resultados. Participaron 780 pacientes; las diferencia en los perfiles entre los hiperfrecuentadores y controles se observaron en los factores de predisposición: sexo, edad, escolaridad; de capacidad: tiempo en acudir a la institución y el medio de transporte que utilizan; y de necesidad: percepción de salud, apoyo social, función familiar, y la presencia de trastornos ansiosos o depresivos. Tener depresión o ansiedad se asoció con la hiperfrecuentación (ORa 1,99; IC 95\%: 1,19-3,31) así como tener un sistema de referencias (ORa 1,61; IC 95\%: 1,01-2,76), la disfunción familiar leve o buena se asoció de manera negativa (ORa: 0,79; IC 95\%: 0,48-0,88) después de ajustar por edad, sexo, etnia e institución prestadora de servicios de salud. Conclusiones. Los pacientes hiperfrecuentadores tienen diferentes perfiles de capacidad, necesidad y predisposición que los controles, los últimos se asociaron de manera independiente a la hiperfrecuentación. En particular, lo trastornos ansioso depresivos duplicaron el riesgo de hiperfrecuentar en atención primaria.
\end{abstract}

Palabras clave: Servicios de salud; Utilización; Ansiedad; Depresión; Atención primaria de la salud (fuente: DeCS BIREME).

\section{PROFILES OF HIGH-FREQUENCY USERS OF PRIMARY CARE SERVICES AND ASSOCIATIONS WITH DEPRESSIVE ANXIETY DISORDERS IN CALI, COLOMBIA}

\begin{abstract}
Objectives. To determine the profiles of highly frequent users of primary care services and the associations of these profiles with depressive anxiety disorders in Cali, Colombia. Materials and Methods. A case-control study, high-frequency cases were defined as those involving patients with a percentile $>75$ with regard to the frequency of spontaneous use of outpatient facilities in the last 12 months; controls were defined as those with a percentile $<25$. A multiple correspondence analysis was used to describe patient profiles, and the influences of depression and anxiety on frequent attendance was determined via logistic regression. Results. Among the 780 participating patients, differences in the profiles among frequent users and controls were related to predisposing factors such as sex, age, and education, capacity factors such as the time required to visit the institution and the means of transport used, and need factors such as health perceptions, social support, family function, and the presence of anxiety or depressive disorders. A depression or anxiety disorder was found to associate positively with frequent attendance (adjusted odds ratio [aOR]: 1.99, 95\% confidence interval [Cl]: 1.19-3.31) and a referral system (aOR: $1.61,95 \% \mathrm{Cl}: 1.01-2.76$ ), but negatively with mild or no family dysfunction (aOR: $0.79 ; 95 \% \mathrm{Cl}: 0.48-0.88$ ) after adjusting for age, sex, ethnicity, and health service-providing institutions. Conclusions. The profiles of high-frequency patients differ from control patients with respect to factors related to capacity, need, and willingness; in particular, the latter were independently associated with frequent attendance. Notably, the presence of an anxious or depressive disorder doubled the risk of highfrequency attendance at a primary care facility
\end{abstract}

Key words: Health services-use; Anxiety; Depression; Primary health care. (source: MeSH NLM)

Grupo de investigación de Salud y Calidad de Vida de la Pontificia Universidad Javeriana. Cali, Colombia.

Grupo de evaluación de servicios de salud de la Caja de Compensación Familiar del Valle del Cauca. Cali, Colombia.

Departamento de Salud Pública y Epidemiología de la Pontificia Universidad Javeriana. Cali, Colombia.

Universidad del Valle. Cali, Colombia

Universidad Icesi. Cali, Colombia.

Grupo de investigación servicios de salud de la Universidad del Valle. Cali, Colombia.

Fundación Valle del Lili. Cali, Colombia.

Medicina Inteligente SAS. Cali, Colombia.

Médico especialista en Medicina familiar, máster en Epidemiologia; ${ }^{\mathrm{b}}$ psicóloga. PhD en Salud Pública; ${ }^{\mathrm{c}}$ médico especialista en otorrinolaringología, máster en Epidemiologia; ${ }^{\mathrm{d}}$ estadístico; ${ }^{\mathrm{e}}$ médico especialista en medicina familiar; ${ }^{\mathrm{f}}$ médico, máster en Salud Pública; ${ }^{\mathrm{g}}$ médico psiquiatra. máster en Salud Pública. Recibido: 19/01/2016 Aprobado: 04/05/2016

Citar como: Rodriguez-Lopez M, Arrivillaga M, Holguín J, León H, Ávila A, Hernández C, Perfil del paciente hiperfrecuentador y su asociación con el trastorno ansioso depresivo en servicios de atención primaria de Cali, Colombia. Rev Peru Med Exp Salud Publica. 2016;33(3):478-88. doi: 10.17843/ rpmesp.2016.333.2335 


\section{INTRODUCCIÓN}

El comportamiento de hiperfrecuentación o uso excesivo de servicios se caracteriza por acudir repetidamente y por iniciativa propia, a las consultas externas y/o de urgencias de los servicios de salud (1). A nivel internacional se ha documentado una prevalencia entre $5-15 \%{ }^{(2)}$ y se ha estimado que 15,4 a $20 \%$ de estas personas continúan hiperfrecuentando al menos durante los siguientes 3 años ${ }^{(3)}$. Estos pacientes tienen mayor riesgo de procedimientos innecesarios, usan más medicamentos y tienen un mayor gasto de bolsillo en comparación con aquellas personas con menor uso de los servicios ${ }^{(4)}$. Se ha propuesto que los grupos en mayor riesgo son las mujeres, los mayores de 50 años, los solteros, y los pacientes con enfermedades crónicas, en particular con trastornos mentales ${ }^{(1,5)}$.

La hiperfrecuentación puede ser explicada desde el modelo de acceso a servicios de Aday y Andersen (6), el cual sugiere que en este comportamiento intervienen factores relacionados con las políticas públicas, las instituciones aseguradoras o prestadoras en salud, el contexto cultural-comunitario-familiar, el profesional de la salud y del paciente ${ }^{(5,6)}$. Con respecto al paciente, el modelo propone que la hiperfrecuentación está determinada por el balance entre los factores de predisposición, capacidad y necesidad. Los primeros son condiciones previas a la ocurrencia del comportamiento que propician su desarrollo. Los factores de capacidad tienen en cuenta el promedio de las capacidades financieras y organizacionales para el uso de servicios. Por su parte, los factores de necesidad se basan en la percepción de daño a la salud, que genera un estado de alerta en el que intervienen la percepción de la severidad de síntomas, de la discapacidad, la susceptibilidad individual y las experiencias clínicas previas.

El paciente hace un balance de los beneficios que le puede traer la utilización de un servicio con las dificultades para alcanzarlo y toma la decisión de la búsqueda o no de la atención. En este sentido, los trastornos ansioso-depresivos podrían modificar la percepción de necesidad y generar el uso de servicios. Algunos estudios en sistemas de salud diferentes al colombiano, han mostrado una asociación positiva entre los trastornos ansioso-depresivos y el uso de servicios ${ }^{(7,8)}$, pero estos estudios no han considerado la influencia de los factores de capacidad y predisposición. En Colombia, las prevalencias de los trastornos mentales son altas ${ }^{(9)}$; sin embargo, algunas barreras de acceso como el uso de copagos o cuotas moderadoras podrían contrarrestar la percepción de necesidad de atención y, por tanto, modular el uso de servicios.
Los estudios nacionales sobre el uso de servicios en atención primaria se orientan a las barreras de acceso ${ }^{(10,11)}$ y no al uso reiterado de los mismos (12). Conocer quiénes son estos pacientes, es condición necesaria para abordar el problema en el contexto colombiano. Los estudios internacionales describen el perfil del hiperfrecuentador con base a las diferencias univariadas $^{(1,4,7)}$; sin embargo, estas no dan cuenta de la presencia simultánea de varias cualidades en un mismo paciente. De igual forma, la influencia independiente de la depresión y la ansiedad en este comportamiento no ha sido, en nuestro conocimiento, abordada en la atención primaria en Colombia. Por esta razón, este estudio tuvo como objetivo identificar el perfil de los hiperfrecuentadores y determinar la influencia de los trastornos mentales comunes (ansiedad-depresión) en este comportamiento.

\section{MATERIALES Y MÉTODOS}

\section{DISEÑO Y POBLACIÓN}

Se realizó un estudio de casos y controles en adultos entre los 18 y 65 años del régimen contributivo. Al régimen contributivo se afilian las personas con vinculación laboral, los pensionados y sus familias. Al momento de requerir una consulta médica externa, el afiliado acude a su institución prestadora de servicios de salud (IPS) y paga un porcentaje del costo de la consulta llamado cuota moderadora, calculados por categoría salarial. Se incluyeron aquellos pacientes que habían acudido al menos una vez a consulta externa de atención primaria durante el 2011, en la ciudad de Cali, Colombia. De manera general, la población de cada IPS vive cerca de la misma, lo que condiciona cierta homogeneidad en el estrato socioeconómico y otros factores relacionados con la comunidad. Se realizó una aleatorización proporcional de casos y controles $( \pm 5 \%$ ) en cada IPS. Todas las IPS siguen protocolos de manejos estandarizados.

Como criterio de inclusión, las personas debían estar afiliadas durante todo el último año a las IPS del estudio. Se excluyeron aquellas con trastorno mental diagnosticado, dada la modificación de la exposición debido al tratamiento psiquiátrico; con fibromialgia o síndrome de intestino irritable, porque la prevalencia de exposición es mayor que en el resto de población; con trastorno neurológico, que imposibilitara la respuesta a la entrevista; postrados o con limitación física para el desplazamiento, por la menor oportunidad de convertirse en caso, y aquellos que estaban afiliados de manera simultánea a una aseguradora privada en salud, dado que estos pacientes suelen consultar los servicios privados. 


\section{SELECCIÓN DE LA MUESTRA Y RECLUTAMIENTO}

El tamaño de la muestra fue calculado en EPIDAT, para un $\mathrm{OR}=2$, una confianza del $95 \%$ y una potencia de $80 \%$; se consideró una prevalencia de exposición en los controles de $6,9 \%$ para el trastorno depresivo ${ }^{(9)} \mathrm{y}$ en los casos de $13 \%$. Se determinó un tamaño de 390 casos e igual número de controles, pero se muestrearon 460 por grupo considerando una no respuesta del $15 \%$. El marco muestral estuvo constituido por los registros institucionales de consultas externas de medicina general en el último año. Se excluyeron las consultas de programas de promoción y prevención. La muestra fue seleccionada de manera aleatoria, previa verificación de los criterios de inclusión y exclusión.

Se llamó telefónicamente a los pacientes para invitarlos a participar en el estudio y solicitarles el consentimiento informado verbal. En ese momento se les explicó en qué consistía la encuesta y el tiempo promedio de respuesta. En caso de detectar síntomas de alarma se brindaron recomendaciones incluyendo la remisión a consulta médica.

\section{VARIABLES DE RESULTADO}

Se definió como caso hiperfrecuentador a todo paciente cuyo número de consultas externas por demanda espontánea fuera $>6$ durante los últimos 12 meses. Este punto de corte fue establecido con base en el valor del percentil 75 de la distribución de consultas en la misma institución. Este punto de corte corresponde con reportes de otras instituciones a nivel nacional, y ha sido utilizado previamente en la literatura ${ }^{(5)}$. Como control concurrente se definió a todo paciente con $\leq 2$ consultas que correspondió con el percentil 25. Como los patrones de utilización pueden variar en el transcurso del año ${ }^{(5)}$, ambos grupos debían tener, al menos, una de las consultas en el último mes. De esta forma, se garantizó que los casos lo siguieran siendo al momento del estudio y que los controles tuvieran un uso de consulta externa anual similar a la esperada para el régimen contributivo ${ }^{(13)}$.

\section{VARIABLES DE EXPOSICIÓN}

Dentro de los factores de predisposición se incluyeron los sociodemográficos, el sistema de referencia evaluado mediante la pregunta: ¿alguna persona le recomienda ir a la IPS cuando presenta algún problema de salud?, y la satisfacción del servicio. Como factores de capacidad se incluyeron los recursos económicos así como el tiempo, medio de transporte y dificultad en llegar a la IPS. Como factores de necesidad se evaluó la ansiedad y/o depresión, mediante el PRIME-MD (Primary Care Evaluation of Mental Disorders). Este instrumento permite identificar trastornos mentales comunes en atención primaria con base en los criterios del DSM-IV (Diagnostic and Statistical Manual of Mental Disorders, Fourth Edition). Tiene una especificidad del $88 \%$ y una sensibilidad del $84 \%$ para los trastornos incluidos en este estudio. Su calificación es dicotómica según la presencia/ausencia del trastorno ${ }^{(14)}$.

Otras variables de necesidad fue la función familiar, obtenida mediante el APGAR familiar, que clasifica la presencia y severidad de la disfunción ${ }^{(15)}$ El apoyo social se midió mediante el cuestionario de soporte social de Duke UNC ${ }^{(16)}$ con sus subescalas afectiva y confidencial. Estos instrumentos han sido validados en castellano y previamente utilizados en investigación. Adicionalmente, se evaluó la percepción de salud, la respuesta frente a un problema de salud y tener algún evento de preocupación y tristeza mediante las preguntas: ¿cómo percibe, en general, su estado de salud en el último año?, ¿qué hace más frecuentemente usted cuando se siente enfermo?, ¿ha tenido alguna situación o problema que le haya generado o genere preocupación, angustia o tristeza?

\section{INSTRUMENTOS Y FUENTES DE INFORMACIÓN}

La fuente primaria fue una entrevista telefónica realizada por un auxiliar de investigación enmascarada frente a la clasificación de caso o control. La información fue digitada en una base de datos en EPIINFO v.3 y procesada en STATA versión 12. Para garantizar la calidad de los datos, el coordinador del proyecto revisó cada uno de los cuestionarios para evaluar su completo diligenciamiento. Para evaluar la calidad de la recogida del dato se verificó aleatoriamente el $5 \%$ de los cuestionarios, contrastándolos con la historia clínica. Adicionalmente, se revisó el $10 \%$ del total de los datos digitalizados, contrastándolos con la información de los cuestionarios para evaluar la calidad en la entrada de datos.

\section{ANÁLISIS ESTADÍSTICO}

Para el análisis descriptivo se utilizaron proporciones que se compararon entre los grupos mediante la prueba de chi cuadrado. Para la determinación del perfil se empleó el análisis de correspondencias múltiples (ACM) para cada tipo de factor (predisposición, capacidad y necesidad). EI ACM es una técnica descriptiva de reducción de dimensiones para variables categóricas $u$ ordinales, que trata de mostrar en dos dimensiones (en nuestro estudio), una realidad multidimensional. Permite resumir la relación entre todas las variables que usualmente se presentan de manera simultánea en un mismo individuo, lo que no se puede apreciar directamente en una tabla de contingencia. Sus resultados suponen que dos 
individuos se parecen si tienen las mismas cualidades (categorías) y si son cercanas estas cualidades en el gráfico bidimensional ${ }^{(17)}$. El número de dimensiones fue seleccionado con aquellas variables con autovalores $>1$ en los gráficos screen-plot. Para la interpretación del perfil se consideró tanto los gráficos de dispersión generados en Excel 2010, así como la contribución de cada modalidad en las tablas de correspondencia (mayores valores absolutos mayor contribución) y su significancia estadística a un nivel alfa de 0,05\%.

Por último, dada la alta correlación entre la ansiedad y la depresión $(r=0,82, p<0,05)$, se generó una variable dicotómica que fue positiva con la presencia de uno o ambos trastornos y se determinó su efecto independiente en la hiperfrecuentación mediante regresión logística múltiple. Se incluyó en todos los modelos la variable IPS. El modelo múltiple se construyó incorporando una variable cada vez al modelo de la ansiedad-depresión (forward selection), con aquellas variables con $p \leq 0,10$ en el análisis univariado y las consideradas confusoras. Desde el punto de vista estadístico se consideró confusora a aquella variable que tuvo un cambio $\geq 10 \%$ entre el OR crudo y el ajustado para ansiedad-depresión. Se seleccionó como modelo final aquel más parsimonioso. La bondad de ajuste se evaluó mediante la prueba de Hosmer-Lemeshow.

\section{CONSIDERACIONES ÉTICAS}

Para mantener la confidencialidad, las encuestas fueron identificadas con un código único el cual se mantuvo en la base de datos. El estudio fue aprobado por el comité de ética de la Pontificia Universidad Javeriana sede Cali y de la institución donde se realizó el estudio.

\section{RESULTADOS}

Participaron 780 personas, de las cuales 408 eran hiperfrecuentadoras $(52,3 \%)$ y 372 controles $(47,7 \%)$ el diagrama de flujo de la selección de la muestra se describe en la Figura 1.

La muestra estuvo constituida principalmente por mujeres (72,7\%), con edad promedio de $40 \pm 12$ años. Respecto a los que participaron en el estudio, los casos que no participaron tenían una edad similar $(45,9 \pm 9,1 ; p=0,09)$ mientras que controles eran casi 6 años más jóvenes $(24,2 \pm 2,2 ; p<0,05)$.

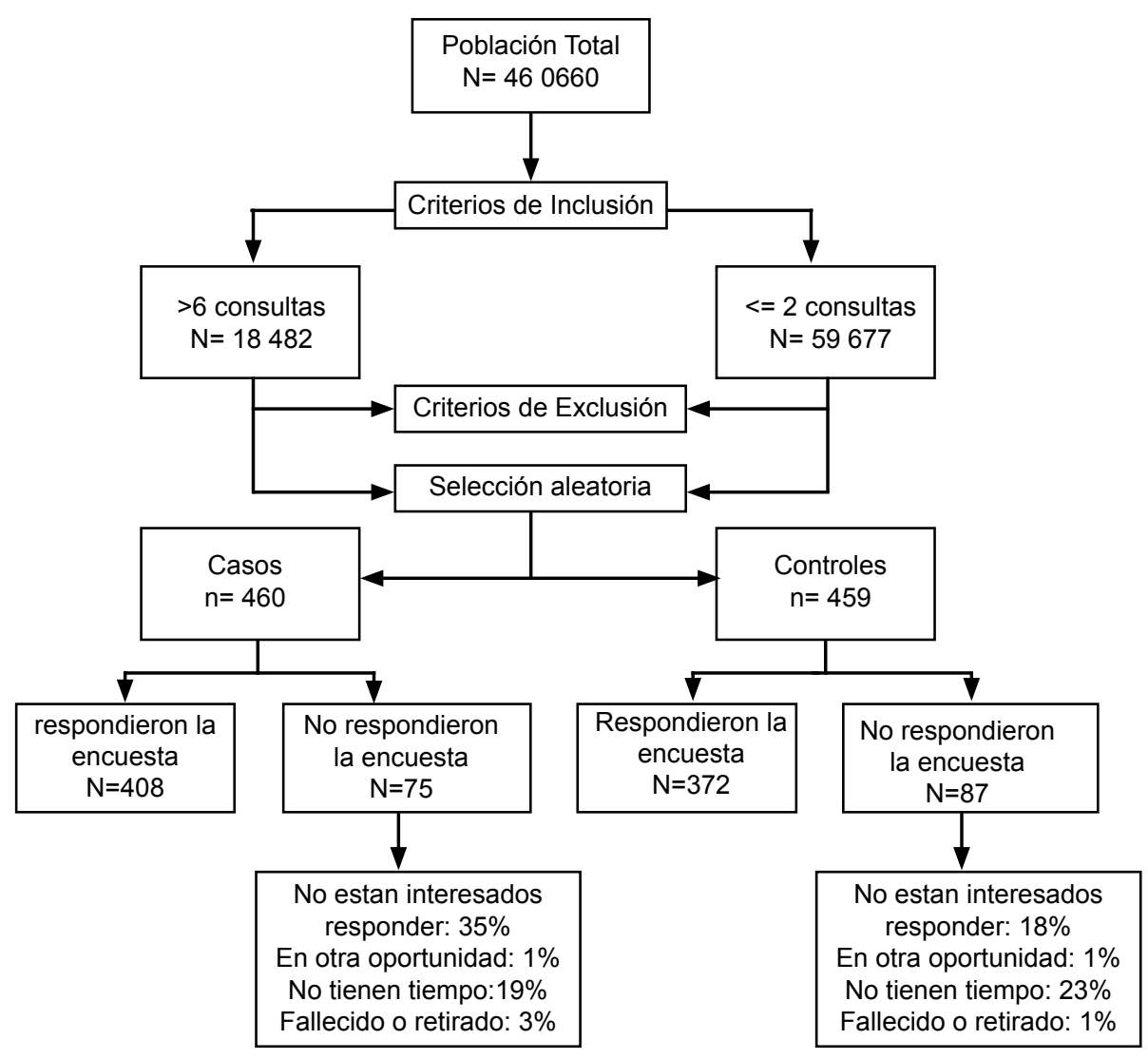

Figura 1. Diagrama de flujo de la selección de la muestra 
La prevalencia de mujeres en los no participante fue menor en casos y mayor en controles respecto a los participantes ( 79,5 y $67,35 \%$, respectivamente).

El coeficiente alfa de Cronbach fue 0,817 para el módulo de depresión (9 ítems), 0,798 para el de ansiedad, 0,862 para el apoyo social, y 0,874 para el APGAR familiar.

En cuanto a los factores de predisposición, se encontraron diferencias significativas entre los grupos en el sexo, edad, etnia, ocupación y escolaridad, y satisfacción del servicio, pero no en el estado civil, vivir solo y contar con un sistema de referencias. En cuanto a los factores de capacidad se observaron diferencias significativas en el rango salarial y el tiempo y medio de transporte para llegar a la IPS, pero no en el gasto económico y la percepción de dificultad para llegar a la misma. Al considerar los factores de necesidad, la percepción de salud, tener alguna preocupación o tristeza, la depresión, ansiedad y el apoyo afectivo fueron diferentes entre los grupos mientras las diferencias fueron no significativas en la respuesta frente a un problema de salud, la ideación suicida, la función familiar y para el apoyo confidencial y afectivo (Tabla 1).

La variabilidad total explicada por los factores de predisposición, capacidad y predisposición fue de $69,19 \%, 72,12 \%$ y $76,10 \%$, respectivamente. En los factores de predisposición, el primer eje explicó un $42 \%$ de la varianza total, y discriminó los hiperfrecuentadores vs controles según los niveles de escolaridad y edad. El segundo eje acumuló un $27,3 \%$, siendo la edad y el sexo las que más contribuyeron a la variabilidad del mismo. La satisfacción del servicio mostró una baja capacidad discriminatoria (Figura 2A, Tabla 2).

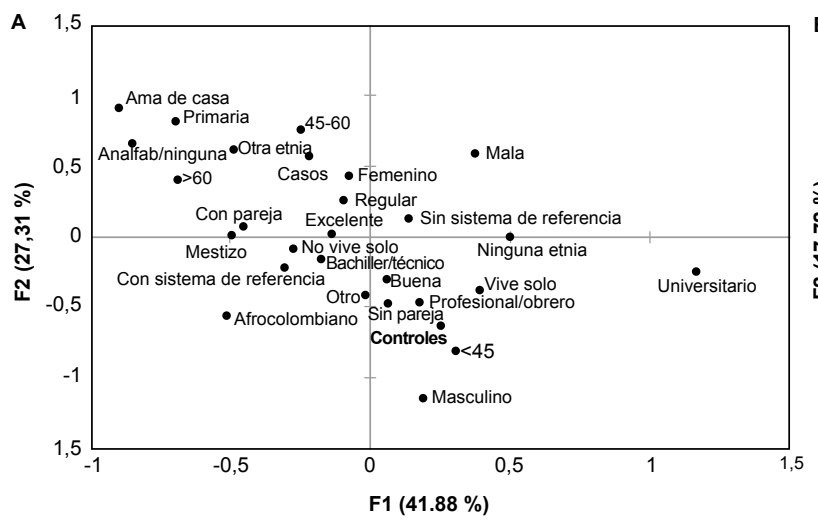

Gráfico simétrico de las variables de predisposición (ejes F1 y F2: 69,19\%)

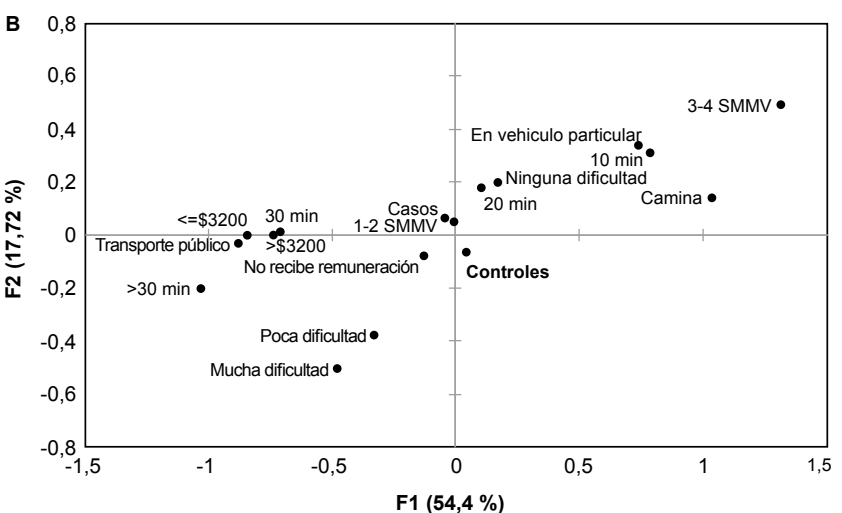

Gráfico simétrico de las variables capacidad (ejes F1 y F2: $72,12 \%$ )

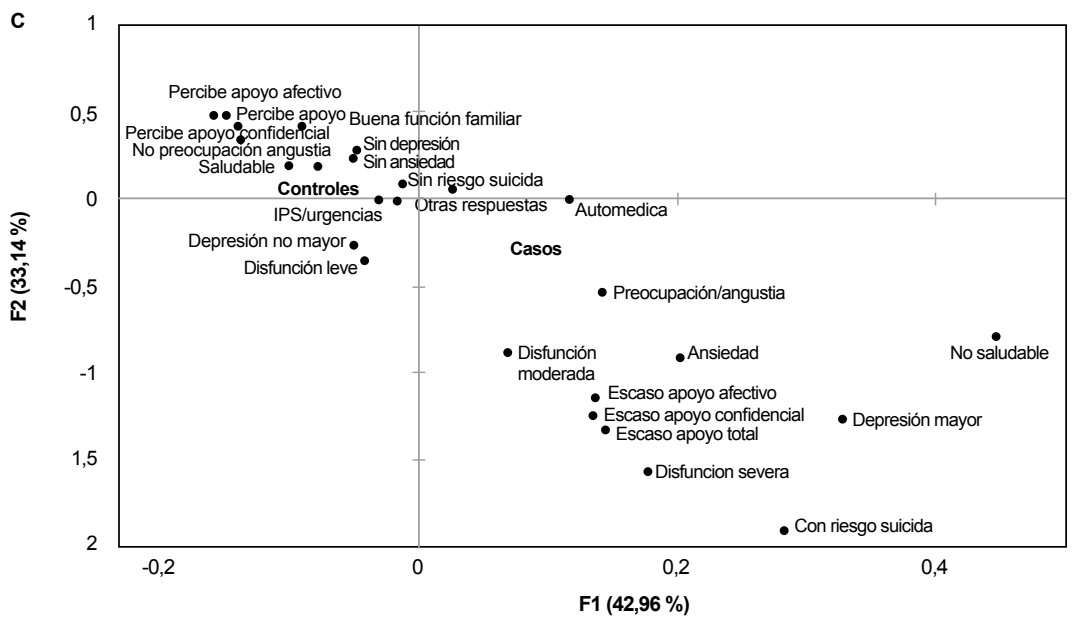

Gráfico simétrico de las variables de necesidad del uso de servicios (ejes F1 y F2: $76,10 \%$ )

Figura 2. Correspondencia múltiple de los factores de A) Predisposición B) Capacidad y C) Necesidad del uso de servicios en hiperfrecuentadores y controles 
Tabla 1. Factores de predisposición, capacidad y necesidad en pacientes hiperfrecuentadores de servicios de atención primaria en Cali, Colombia

\begin{tabular}{|c|c|c|c|c|}
\hline Características & & $\begin{array}{c}\text { Hiperfrecuentador } \\
\mathrm{n}(\%)\end{array}$ & $\begin{array}{c}\text { Control } \\
\mathrm{n}(\%)\end{array}$ & Valor $p$ \\
\hline \multicolumn{5}{|l|}{ Predisposición } \\
\hline Sexo & $\begin{array}{l}\text { Femenino } \\
\text { Masculino }\end{array}$ & $\begin{array}{r}380(93,1) \\
28(6,9)\end{array}$ & $\begin{array}{l}187(50,3) \\
185(49,7)\end{array}$ & $<0,001$ \\
\hline & $<45$ & $40(9,8)$ & $332(89,2)$ & $<0,001$ \\
\hline Edad agrupada, años & $\begin{array}{l}45-60 \\
>\text { de } 60\end{array}$ & $\begin{array}{r}359(88,0) \\
9(2,2)\end{array}$ & $\begin{array}{r}30(8,1) \\
9(2,4)\end{array}$ & \\
\hline Escolaridad $(n=727)$ & $\begin{array}{l}\text { Analfabeto/ninguna } \\
\text { Primaria } \\
\text { Bachiller/técnico } \\
\text { Universitario }\end{array}$ & $\begin{array}{r}6(1,5) \\
85(20,8) \\
255(62,5) \\
36(8,8)\end{array}$ & $\begin{array}{r}5(1,3) \\
29(7,8) \\
248(66,7) \\
63(16,9)\end{array}$ & $<0,001$ \\
\hline \multirow{2}{*}{ Estado civil $(\mathrm{n}=687)$} & Sin Pareja & $111(27,2)$ & $128(34,4)$ & 0,083 \\
\hline & Con Pareja & $248(60,8)$ & $200(53,8)$ & \\
\hline Ocupación actual (n=732) & $\begin{array}{l}\text { Ama de casa } \\
\text { Profesional/obrero } \\
\text { Otra }\end{array}$ & $\begin{array}{r}146(35,8) \\
185(45,3) \\
42(10,3)\end{array}$ & $\begin{array}{r}42(11,3) \\
253(68,0) \\
64(17,2)\end{array}$ & $<0,001$ \\
\hline Etnia $(n=779)$ & $\begin{array}{l}\text { Afrocolombiano } \\
\text { Mestizo } \\
\text { Otra } \\
\text { Ninguno }\end{array}$ & $\begin{array}{r}40(9,8) \\
159(39,0) \\
44(10,8) \\
165(40,4)\end{array}$ & $\begin{array}{r}34(9,1) \\
101(27,2) \\
20(5,4) \\
216(58,1)\end{array}$ & $<0,001$ \\
\hline Vivir solo $(n=698)$ & $\begin{array}{l}\text { No } \\
\text { Sí }\end{array}$ & $\begin{array}{r}356(87,3) \\
10(2,5)\end{array}$ & $\begin{array}{r}324(87,1) \\
8(2,2)\end{array}$ & 0,788 \\
\hline Sistema de referencia $(n=771)$ & $\begin{array}{l}\text { No } \\
\text { Sí }\end{array}$ & $\begin{array}{l}267(65,4) \\
139(34,1)\end{array}$ & $\begin{array}{l}253(69,3) \\
112(30,7)\end{array}$ & 0,293 \\
\hline Satisfacción del servicio $(n=778)$ & $\begin{array}{l}\text { Excelente } \\
\text { Bueno } \\
\text { Regular } \\
\text { Malo }\end{array}$ & $\begin{array}{r}60(14,7) \\
160(39,2) \\
154(37,7) \\
33(8,1)\end{array}$ & $\begin{array}{r}51(13,7) \\
186(50,0) \\
112(30,1) \\
22(5,9)\end{array}$ & 0,043 \\
\hline \multicolumn{5}{|l|}{ Capacidad } \\
\hline Rango salarial, SMMV (n=692) & $\begin{array}{l}\text { No responde } \\
\text { No recibe remuneración } \\
\text { 1-2 SMMV } \\
2-4 \text { SMMV }\end{array}$ & $\begin{array}{r}81(19,9) \\
189(46,3) \\
134(32,8) \\
4(1,0)\end{array}$ & $\begin{array}{r}88(23,3) \\
94(25,3) \\
186(50,0) \\
4(1,1)\end{array}$ & $<0,001$ \\
\hline Medio de transporte a la IPS $(n=773)$ & $\begin{array}{l}\text { En vehículo particular } \\
\text { Transporte público } \\
\text { Camina }\end{array}$ & $\begin{array}{r}47(11,5) \\
212(52,0) \\
149(36,5)\end{array}$ & $\begin{array}{r}94(25,3) \\
191(51,3) \\
80(21,5)\end{array}$ & $<0,001$ \\
\hline Gasto de transporte, pesos colombianos $(n=441)$ & $\begin{array}{l}<=3200 \\
>3200\end{array}$ & $\begin{array}{r}169(72,2) \\
65(27,8)\end{array}$ & $\begin{array}{r}145(70,0) \\
62(30,0)\end{array}$ & 0,615 \\
\hline Tiempo en llegar a la IPS, minutos & & $23,9(16,8)$ & $20,6(12,9)$ & 0,019 \\
\hline Dificultad en llegar a la IPS $(n=779)$ & $\begin{array}{l}\text { Ninguna } \\
\text { Poca } \\
\text { Mucha }\end{array}$ & $\begin{array}{r}266(65,2) \\
97(23,8) \\
44(10,8)\end{array}$ & $\begin{array}{r}261(70,2) \\
86(23,1) \\
25(6,7)\end{array}$ & 0,152 \\
\hline \multicolumn{5}{|l|}{ Necesidad } \\
\hline Percepción de salud & $\begin{array}{l}\text { Saludable } \\
\text { No saludable }\end{array}$ & $\begin{array}{l}302(74,0) \\
106(26,0)\end{array}$ & $\begin{array}{r}335(90,1) \\
37(9,9)\end{array}$ & $<0,001$ \\
\hline Respuesta a un problema de salud & $\begin{array}{l}\text { IPS/urgencias } \\
\text { Se automedica } \\
\text { Otras }\end{array}$ & $\begin{array}{r}283(69,4) \\
50(12,3) \\
75(18,4)\end{array}$ & $\begin{array}{r}248(66,8) \\
52(14,0) \\
72(19,4)\end{array}$ & 0,688 \\
\hline Preocupación/ angustia $(n=771)$ & $\begin{array}{l}\text { No } \\
\text { Sí }\end{array}$ & $\begin{array}{l}205(50,7) \\
199(49,3)\end{array}$ & $\begin{array}{r}271(73,8) \\
96(26,2)\end{array}$ & $<0,001$ \\
\hline Depresión & $\begin{array}{l}\text { Sin depresión } \\
\text { Depresión no mayor } \\
\text { Depresión mayor }\end{array}$ & $\begin{array}{r}270(66,2) \\
61(15,0) \\
77(18,9)\end{array}$ & $\begin{array}{r}325(87,4) \\
26(7,0) \\
21(5,6)\end{array}$ & $<0,001$ \\
\hline Ideación suicida & $\begin{array}{l}\text { No } \\
\text { Sí }\end{array}$ & $\begin{array}{r}21(5,1) \\
387(94,9)\end{array}$ & $\begin{array}{r}10(2,7) \\
362(97,3)\end{array}$ & 0,079 \\
\hline Ansiedad & No & $\begin{array}{l}298(73,0) \\
110(27,0)\end{array}$ & $\begin{array}{r}331(89,0) \\
41(11,0)\end{array}$ & $<0,001$ \\
\hline Función familiar & $\begin{array}{l}\text { Buena } \\
\text { Disfunción leve } \\
\text { Disfunción moderada } \\
\text { Disfunción severa }\end{array}$ & $\begin{array}{r}256(63,2) \\
77(19,0) \\
41(10,1) \\
31(7,7)\end{array}$ & $\begin{array}{r}241(65,0) \\
72(19,5) \\
36(9,7) \\
22(5,9)\end{array}$ & 0,805 \\
\hline \multicolumn{5}{|l|}{ Apoyo social } \\
\hline Apoyo total $(n=776)$ & $\begin{array}{l}\text { Escaso } \\
\text { Percibe }\end{array}$ & $\begin{array}{l}116(28,6) \\
289(71,4)\end{array}$ & $\begin{array}{r}92(24,8) \\
279(75,2)\end{array}$ & 0,227 \\
\hline Apoyo confidencial $(n=776)$ & $\begin{array}{l}\text { Escaso } \\
\text { Percibe }\end{array}$ & $\begin{array}{r}101(24,9) \\
304(75,1)\end{array}$ & $\begin{array}{r}95(25,6) \\
276(74,4)\end{array}$ & 0,800 \\
\hline Apoyo afectivo $(n=776)$ & $\begin{array}{l}\text { Escaso } \\
\text { Percibe }\end{array}$ & $\begin{array}{l}136(33,6) \\
269(66,4)\end{array}$ & $\begin{array}{r}96(25,9) \\
275(74,1)\end{array}$ & 0,024 \\
\hline
\end{tabular}

IPS: Institución Prestadora de Servicios de Salud, SMMV: Salario Mínimo Mensual Vigente. 
Tabla 2. Valores de las modalidades del análisis de correspondencia múltiple de los factores de predisposición

\begin{tabular}{|c|c|c|}
\hline Modalidades & Eje 1 & Eje 2 \\
\hline Sexo & & $19,641^{*}$ \\
\hline Femenino & $-3,248^{*}$ & \\
\hline Masculino & $3,248^{*}$ & $-19,641^{*}$ \\
\hline \multicolumn{3}{|l|}{ Edad } \\
\hline$<45$ & $7,822^{*}$ & $-20,660^{*}$ \\
\hline $45-60$ & $-6,885^{\star}$ & $20,007^{*}$ \\
\hline$>60$ & $-2,945^{\star}$ & 1707 \\
\hline Escolaridad & $-2,825^{\star}$ & $2,188^{*}$ \\
\hline \multicolumn{3}{|l|}{ Analfabeta/ninguna } \\
\hline Primaria & $-8,008^{*}$ & $9,451^{*}$ \\
\hline Bachiller/técnico & $-6,578^{*}$ & $-6,116^{*}$ \\
\hline Universitario & $12,422^{*}$ & $-2,696^{*}$ \\
\hline Estado civil & 1223 & $-8,862^{*}$ \\
\hline \multicolumn{3}{|l|}{ Sin Pareja } \\
\hline Con Pareja & $-14,565^{\star}$ & $2,279^{*}$ \\
\hline Ocupación & $-14,062^{*}$ & $14,342^{*}$ \\
\hline \multicolumn{3}{|l|}{ Ama de casa } \\
\hline Profesional/obrero & $5,674^{*}$ & $-14,739^{*}$ \\
\hline Otro & $-0,166$ & $-4,628^{*}$ \\
\hline Etnia & $-4,647^{*}$ & $-5,236^{*}$ \\
\hline \multicolumn{3}{|l|}{ Afrocolombiano } \\
\hline Mestizo & $-9,703^{*}$ & 0,162 \\
\hline Otro & $-4,064^{*}$ & $5,120^{*}$ \\
\hline Ninguno & $13,754^{*}$ & $-0,020$ \\
\hline Vive solo & $-19,857^{*}$ & $-6,770^{*}$ \\
\hline \multicolumn{3}{|l|}{ No } \\
\hline Sí & 1673 & -1813 \\
\hline Sistema de referencia & $5,323^{*}$ & $4,996^{*}$ \\
\hline \multicolumn{3}{|l|}{ No } \\
\hline Sí & $-5,853^{*}$ & $-4,402^{*}$ \\
\hline Satisfacción del servicio & $2,875^{\star}$ & $4,502^{*}$ \\
\hline \multicolumn{3}{|l|}{ Malo } \\
\hline Regular & -1839 & $4,990^{*}$ \\
\hline Bueno & 1492 & $-7,048^{*}$ \\
\hline Excelente & -1524 & 0,181 \\
\hline \multicolumn{3}{|l|}{ Población } \\
\hline Casos & $-6,716^{*}$ & $16,772^{*}$ \\
\hline Controles & $6,716^{*}$ & $-16,772^{*}$ \\
\hline
\end{tabular}

${ }^{*} p<0,05$

En cuanto a los factores de capacidad, el primer eje explicó un $54,4 \%$ de la inercia total y discriminó los
Tabla 3. Valores de las modalidades del análisis de correspondencia múltiple de los factores de capacidad

\begin{tabular}{|c|c|c|}
\hline Modalidades & Eje 1 & Eje 2 \\
\hline \multicolumn{3}{|l|}{ Rango salarial } \\
\hline No responde & $2,322^{*}$ & 0,502 \\
\hline No recibe remuneración & $-2,653^{*}$ & $-1,822$ \\
\hline 1-2 SMMV & $-0,115$ & 1,077 \\
\hline 3-4 SMMV & $3,727^{*}$ & 1,385 \\
\hline \multicolumn{3}{|l|}{ Medio de transporte } \\
\hline En vehículo particular & $9,696^{*}$ & $4,418^{*}$ \\
\hline Transporte público & $-25,372^{*}$ & $-0,987$ \\
\hline Camina & $18,743^{*}$ & $2,502^{*}$ \\
\hline \multicolumn{3}{|l|}{ Gasto de transporte } \\
\hline$\leq \$ 3200$ & $-19,179^{*}$ & $-0,090$ \\
\hline$>\$ 3200$ & $-9,014^{*}$ & $-0,006$ \\
\hline \multicolumn{3}{|l|}{ Tiempo en llegar a la IPS } \\
\hline $10 \min$ & $13,370^{*}$ & $5,182^{*}$ \\
\hline $20 \min$ & $2,342^{*}$ & $3,878^{*}$ \\
\hline $30 \mathrm{~min}$ & $-9,109^{*}$ & 0,115 \\
\hline$>30 \min$ & $-12,028^{*}$ & $-2,384^{*}$ \\
\hline \multicolumn{3}{|l|}{ Dificultad en llegar a la IPS } \\
\hline Ninguna dificultad & $6,936^{*}$ & $7,934^{*}$ \\
\hline Poca dificultad & $-5,081^{*}$ & $-5,860^{*}$ \\
\hline Mucha dificultad & $-4,145^{*}$ & $-4,424^{*}$ \\
\hline \multicolumn{3}{|l|}{ Población } \\
\hline Casos & $-1,231$ & 1,830 \\
\hline Controles & 1,231 & $-1,830$ \\
\hline
\end{tabular}

${ }^{*} p<0,05$

IPS: Institución Prestadora de Servicios de Salud, SMMV: Salario Mínimo Mensual Vigente.

grupos de acuerdo al medio de transporte y el tiempo en llegar a la IPS.

El segundo eje explicó un 17,7\% y discriminó los grupos por la dificultad en llegar a la IPS. El rango salarial contribuyó poco en ambos ejes (Figura 2B, Tabla 3).

En cuanto a los factores de necesidad, el primer eje explicó un $43 \%$ de la inercia total y discriminó los grupos según el apoyo percibido y la percepción de salud. El segundo eje explicó un 33\%. La ansiedad, la depresión y el funcionamiento familiar discriminaron bien los grupos en este eje. La respuesta frente a un problema de salud contribuyó poco a la discriminación (Figura 2C, Tabla 4)

La presencia de depresión-ansiedad se asoció de manera positiva con la hiperfrecuentación (ORa 1,99; 
Tabla 4. Valores de las modalidades del análisis de correspondencia múltiple de los factores de necesidad

\begin{tabular}{|c|c|c|}
\hline Modalidades & Eje 1 & Eje 2 \\
\hline \multicolumn{3}{|l|}{ Percepción de salud } \\
\hline Saludable & $-5,920^{*}$ & $10,621^{*}$ \\
\hline No saludable & $5,920^{*}$ & $-10,621^{*}$ \\
\hline \multicolumn{3}{|l|}{$\begin{array}{l}\text { Respuesta a un problema de } \\
\text { salud }\end{array}$} \\
\hline IPS/urgencias & $-1,231$ & $-0,600$ \\
\hline Automedica & 1,269 & $-0,135$ \\
\hline Otras & 0,373 & 0,831 \\
\hline \multicolumn{3}{|l|}{ Evento preocupación } \\
\hline No & $-4,760^{*}$ & $11,168^{*}$ \\
\hline Sí & $3,110^{*}$ & $-11,809^{*}$ \\
\hline \multicolumn{3}{|l|}{ Depresión } \\
\hline Sin depresión & $-2,354^{*}$ & $12,427^{*}$ \\
\hline No mayor & $-0,480$ & $-2,671^{*}$ \\
\hline Mayor & $3,477^{*}$ & $-13,412^{*}$ \\
\hline \multicolumn{3}{|l|}{ Ideación suicida } \\
\hline No & $-1,610$ & $10,837^{*}$ \\
\hline Sí & 1,610 & $-10,837^{*}$ \\
\hline \multicolumn{3}{|l|}{ Ansiedad } \\
\hline No & $-2,776^{*}$ & $12,628^{*}$ \\
\hline Sí & $2,776^{*}$ & $-12,628^{*}$ \\
\hline \multicolumn{3}{|l|}{ Función familiar } \\
\hline Buena & $-3,307^{*}$ & $14,910^{*}$ \\
\hline Disfunción leve & $-0,562$ & $-4,978^{*}$ \\
\hline Disfunción moderada & 0,646 & $-8,203^{*}$ \\
\hline Disfunción severa & 1,338 & $-11,811^{*}$ \\
\hline \multicolumn{3}{|l|}{ Apoyo social } \\
\hline Escaso apoyo total & $2,453^{*}$ & $-22,417^{*}$ \\
\hline Percibe apoyo & $-6,746^{*}$ & $21,498^{*}$ \\
\hline Escaso apoyo confidencial & $2,199^{*}$ & $-20,261^{*}$ \\
\hline Percibe apoyo confidencial & $-6,574^{*}$ & $19,327^{*}$ \\
\hline Escaso apoyo afectivo & $2,503^{*}$ & $-20,850^{*}$ \\
\hline Percibe apoyo afectivo & $-6,663^{*}$ & $19,989^{*}$ \\
\hline \multicolumn{3}{|l|}{ Población } \\
\hline Casos & $2,059^{*}$ & $-4,513^{*}$ \\
\hline Controles & $-2,059^{*}$ & $4,513^{*}$ \\
\hline
\end{tabular}

${ }^{*} p<0,05$

IC 95\%:1,19-3,31; $p=0,008)$, después de ajustar por covariables. También se asociaron positivamente el incremento anual de la edad (ORa 1,16; IC 95\%: 1,14$1,19 ; p<0,001)$, contar con un sistema de referencia (ORa 1,61; IC 95\%:1,01-2,76; $p=0,047$ ), mientras que el sexo masculino (ORa 0,12; IC 95\%: 0,07-0,22; $p<0,001$ ), las etnias no afrocolombianas (ORa 0,65; IC 95\%: 0,460,$96 ; p=0,015$ ), y la buena función familiar (ORa 0,79; IC 95\%: 0,48-0,88; $p=0,005)$ se asociaron de manera negativa con la hiperfrecuentación (Tabla 5).
Tabla 5. Factores asociados de manera independiente a la hiperfrecuentación de servicios en una IPS de baja complejidad de Cali

\begin{tabular}{|c|c|c|}
\hline Característica & $\begin{array}{l}\text { OR crudo } \\
\text { (IC 95\%) }\end{array}$ & $\begin{array}{l}\text { OR ajustado } \\
\text { (IC } 95 \%)^{*}\end{array}$ \\
\hline \multicolumn{3}{|l|}{ Depresión/ansiedad } \\
\hline No & Ref & Ref \\
\hline Sí & $3,46(2,48-4,82)$ & $1,99(1,19-3,31)$ \\
\hline \multicolumn{3}{|l|}{ Sexo } \\
\hline Femenino & Ref & Ref \\
\hline Masculino & $0,08(0,05-0,12)$ & $0,12(0,07-0,22)$ \\
\hline Edad en años ${ }^{* *}$ & $1,18(1,16-1,21)$ & $1,16(1,14-1,19)$ \\
\hline \multicolumn{3}{|l|}{ Etnia } \\
\hline Afrocolombiana & Ref & Ref \\
\hline Otras & $0,76(0,66-0,87)$ & $0,65(0,46-0,92)$ \\
\hline \multicolumn{3}{|l|}{$\begin{array}{l}\text { Sistema de } \\
\text { referencia }\end{array}$} \\
\hline No & Ref & Ref \\
\hline Sí & $1,20(0,89-1,63)$ & $1,61(1,01-2,76)$ \\
\hline \multicolumn{3}{|l|}{$\begin{array}{l}\text { Preocupación o } \\
\text { angustia }\end{array}$} \\
\hline No & Ref & Ref \\
\hline Sí & $2,73(2,01-3,70)$ & $1,55(0,95-2,54)$ \\
\hline \multicolumn{3}{|l|}{ Función familiar } \\
\hline $\begin{array}{l}\text { Disfunción } \\
\text { moderada- } \\
\text { severa }\end{array}$ & Ref & Ref \\
\hline $\begin{array}{l}\text { Disfunción leve } \\
\text { o buena función }\end{array}$ & $1,07(0,89-1,28)$ & $0,79(0,48-0,88)$ \\
\hline
\end{tabular}

*Datos ajustados por las variables incluidas en el modelo y por IPS ** El coeficiente de regresión de la variable edad expresa el incremento promedio del riesgo por cada año adicional

\section{DISCUSIÓN}

En nuestro conocimiento, este es el primer estudio en Colombia sobre la hiperfrecuentación en atención primaria y sus resultados sugieren que los hiperfrecuentadores superan las barreras de acceso y acuden a las consultas, sin importar el gasto o dificultad en llegar a los servicios. EI ACM mostró que los factores del modelo teórico discriminan bien los grupos, pero fueron los factores de necesidad los que explicaron el mayor porcentaje de la varianza. En particular, las personas con ansiedad o depresión tuvieron el doble de oportunidad de hiperfrecuentar en comparación con los que no tenían estos trastornos.

Los hiperfrecuentadores se caracterizaron por ser en su mayoría mujeres mestizas o afrocolombianas entre 45-60 años, y están casadas o en unión libre a quienes les recomiendan asistir a la IPS frente a un problema de salud. Un pequeño subgrupo son $>60$ años, amas de casa y tienen escolaridad primaria o ninguna. La mayoría no reciben remuneración o máximo de 1-2 salarios 
mínimos, utilizan trasporte público y demoran $\geq 30$ min en llegar a la IPS. Algunos consideran difícil llegar al sitio de atención. Refieren estar preocupados o tristes, tienen ansiedad, escaso apoyo social y algunas presentan depresión mayor y disfunción familiar moderada-severa. Por su parte, la mayoría de los controles eran hombres menores de 45 años, sin pareja estable viven solos y no se consideran de las etnias exploradas, la mayoría tienen nivel universitario y son profesionales $u$ obreros, tienen buena percepción del servicio al cual llegan caminando o en vehículo particular y demoran de 10 a $20 \mathrm{~min}$, por lo cual no perciben dificultad para asistir. Se consideran saludables y con buena función familiar, no tienen ansiedad ni depresión ni riesgo suicida, ni eventos que los preocupen y reciben todas las formas de apoyo social.

La mayoría de los estudios describen los perfiles de los casos mediante las diferencias univariadas ${ }^{(4,18,19)}$, pero estas diferencias no reflejan cómo se relacionan las categorías de múltiples variables en un mismo individuo. Una de las ventajas del ACM se evidenció cuando una cualidad de una variable tuvo distribuciones similares en los grupos, pero el ACM permitió asociar dicha cualidad a un grupo considerando la relación con las cualidades de otras variables. Un ejemplo fue la mayor proporción de individuos con transporte público en toda la población, pero al analizar esta variable en su relación con las otras, se observó una mayor contribución al perfil del grupo hiperfrecuentador (Figura 2). El ACM visibilizó la heterogeneidad que pude existir dentro de un mismo grupo, por ejemplo, cuando identificó un subgrupo de casos mayores de 60 años, con factores de predisposición diferentes al resto del grupo. Así mismo, las pruebas estadísticas para variables ordinales no identifican cuál de los órdenes representa la diferencia entre los grupos, como la disfunción familiar que no mostró diferencias en el análisis univariado, pero se pudo visualizar una gran proximidad entre la buena función familiar, percibir apoyo, sentirse saludable, y no tener ningún evento de preocupación o angustia en los controles.

Nuestros resultados son consistentes con la literatura que reporta mayor prevalencia en mujeres y en edades más avanzadas ${ }^{(2,20,21)}$. De otra parte, estos resultados sugieren que en la retroalimentación de la experiencia de uso podría tener mayor valor la satisfacción de la necesidad que la satisfacción con el servicio, dado que esta última no contribuyó al perfil de los hiperfrecuentadores. Estos resultados difieren de aquellos que reportan una asociación entre la buena satisfacción del servicio y el mayor uso de los mismos ${ }^{(21,22)}$, tal vez por las diferencias culturales entre las poblaciones. De manera interesante, las características tradicionalmente relacionadas con las barreras de acceso como el bajo nivel educativo, bajos ingresos, no tener medio de transporte propio y requerir un mayor tiempo para llegar a la IPS (23), parecen ser superadas por los factores de necesidad de los hiperfrecuentadores en nuestro contexto. De igual forma, los resultados coinciden con aquellos que proponen que las redes de apoyo familiares y sociales podrían regular el uso de servicios ${ }^{(24,25)}$. En nuestra población, tanto el apoyo social como la función familiar discriminaron a los casos de los controles. Es posible que las personas, cuando se sienten enfermas y sin apoyo, puedan desarrollar trastornos ansioso-depresivos, pero el análisis múltiple sugiere un efecto independiente de la disfunción familiar en la hiperfrecuentación.

En cuanto a la asociación de la ansiedad-depresión con la hiperfrecuentación, nuestros resultados son similares a reportes previos internacionales ${ }^{(1,8,26,27)}$. Así mismo, una alta prevalencia de estos trastornos se observó en casos de la misma institución en $2007^{(28)}$, aunque aquel estudio no tuvo grupo control para determinar medidas de riesgo. Es posible que la presencia de ansiedaddepresión produzca cambios en la autopercepción de salud y que al percibirse muy enfermos, estos pacientes decidan superar sus barreras de acceso. De otra parte, se sabe que la ansiedad puede ocasionar síntomas físicos como dolor, fatiga, y palpitaciones y que la depresión puede acompañarse de astenia, adinamia o insomnio (29). Los hiperfrecuentadores usualmente acuden con estos síntomas ${ }^{(23)}$, por lo cual, si el médico no considera la posibilidad de un trastorno mental subyacente, la necesidad que da origen a la atención no es resuelta del todo, perpetuándose el ciclo de atención.

Este es uno de los estudios con mayor tamaño de muestra en el tema, y el método estadístico empleado asegura la descripción del perfil considerando la presencia simultánea de varias características en un mismo paciente. Sin embargo, también tiene algunas limitaciones. En primer lugar, su objetivo fue describir el perfil de los pacientes ubicados en ambos extremos de la curva de uso de servicios para garantizar la correcta clasificación de la variable resultado, por ello se excluyeron aquellos que consultaron entre 3-6 veces; por tanto, no es posible establecer una relación dosisrepuesta. Los casos que participaron tenían mayor proporción de mujeres que los no participantes, pero el sesgo que esto podría producir es compensado, al menos parcialmente, porque los controles que participaron tenían mayor edad que los no participantes. De otra parte, por ser una población del régimen contributivo, limita que sus resultados se extrapolen directamente a poblaciones y servicios diferentes como a las consultas de carácter preventivo, a las cuales usualmente acceden personas con niveles socioeconómicos más altos. Finalmente, el diseño del estudio no permite determinar si la ansiedad-depresión se relaciona más con la cronicidad de la hiperfrecuentación o con su incidencia.

En conclusión las posibles barreras de acceso (factores de capacidad) parecen ser superadas por los factores 
de predisposición y necesidad en los hiperfrecuentadores. Los factores de necesidad son los que mejor contribuyen al perfil de los casos. Dentro de estos, los trastornos mentales ansioso-depresivos se asociaron de manera independiente al uso de servicios. Estos resultados apuntan al tamizaje de los trastornos mentales comunes en servicios de atención primaria, particularmente en hiperfrecuentadoras, mujeres mayores de 45 años, las cuales también podrían requerir una evaluación e intervención en familiar, como posibles estrategias moduladoras del uso de servicios de salud en cuidado primario.

Agradecimientos: A Harold Steven Basallo, monitor de investigaciones de la Pontificia Universidad Javeriana sede Cali por su participación en la recolección de la información y captura de datos. A Martha Lucla Ramos por facilitar el acceso a la información y la logística para realizar el estudio.

Contribución de autores: MRL participo en la concepción y diseño del estudio; recolección de resultados; análisis e interpretación de datos; redacción del artículo y aprobación de la versión final. MA, JH, AA, CH y HGR participaron en la interpretación de datos; redacción del artículo y aprobación de la versión final. HL participo en el análisis estadístico; redacción del artículo; interpretación de datos; redacción del artículo y aprobación de la versión final.

Fuentes de financiamiento: el presente estudio recibió financiación de la Pontificia Universidad Javeriana, Cali.

Conflictos de interés: los autores declaran no tener conflictos de interés en la publicación de este artículo.

\section{REFERENCIAS BIBLIOGRÁFICAS}

1. Vedsted P, Christensen MB. Frequent attenders in general practice care: A literature review with special reference to methodological considerations. Public Health. 2005;119(2):118-37.

2. Smits FT, Brouwer HJ, ter Riet G, van Weert HC. Epidemiology of frequent attenders: a 3-year historic cohort study comparing attendance, morbidity and prescriptions of oneyear and persistent frequent attender. BMC Public Health. 2009;9:36. doi: $10.1186 / 1471-2458-9-36$

3. Smits FT, Brouwer HJ, van Weert HCP, Schene AH, ter Riet G. Predictability of persistent frequent attendance: a historic 3-year cohort study. Br J Gen Pract. 2009;59(559):e44-50. doi: 10.3399/bjgp09X395120.

4. Vedsted P, Christensen MB, Sorensen HT, Fink P, Olesen F. Special status consultation for frequent attenders. Who are the candidates?? J Public Health Med. 2002;24(1):53-7.

5. Rodriguez M. Abordaje del paciente hiperfrecuentador de servicios en atención primaria: un acercamiento desde la teoría. Rev Gerenc Polit Salud. 2012;11(22):43-55.

6. Aday LA, Andersen R. Framework for the study of access to medical care Health Services Research. Health Serv Res. 1974;9(3):208-20.

7. Vedsted P, Fink P, Sørensen HT, Olesen F. Physical, mental and social factors associated with frequent attendance in Danish general practice. A populationbased cross-sectional study. Soc Sci Med. 2004;59(4):813-23.

8. Smits FT, Brouwer HJ, Zwinderman $\mathrm{AH}$, Mohrs J, Schene AH, van
Weert HC, et al. Why do they keep coming back? Psychosocial etiology of persistence of frequent attendance in primary care: a prospective cohort study. J Psychosom Res. 2014;77(6):492-503. doi: 10.1016/j.jpsychores.2014.08.003.

9. Ministerio de la Protección Social, República de Colombia. Estudio Nacional de Salud Mental Colombia 2003 [Internet].Cali:MPS;2005 [citado el 12 de octubre de 2014]. Disponible en: https://www.minsalud.gov.co/ Documentos\%20y\%20Publicaciones/ ESTUDIO\%20NACIONAL\%20 DE\%20SALUD\%20MENTAL\%20 EN\%20COLOMBIA.pdf

10. Vargas J, Molina G. Acceso a los servicios de salud en seis ciudades de Colombia: limitaciones y consecuencias. Rev Fac Nac Salud Pública 2009;27(2):121-30.

11. Mejia-Mejia A, Sanchez-Gandur AF, Tamayo-Ramirez JC. [Equity in access to health-services in Antioquia, Colombia]. Rev Salud Publica (Bogota). 2007;9(1):26-38. [Article in Spanish]

12. Smits FT, Brouwer HJ, Zwinderman AH, Mohrs J, Smeets HM, Bosmans JE, et al. Morbidity and doctor characteristics only partly explain the substantial healthcare expenditures of frequent attenders: a record linkage study between patient data and reimbursements data.. BMC Fam Pract. 2013;14:138. doi: 10.1186/14712296-14-138.

13. Bolívar MC, Alfonzo EA, Hurtado G, Guerrero H, Aldea G, Torres G. Estudio de la suficiencia y de los mecanismos de ajuste de riesgo para el cálculo de la
Unidad de Pago por Capitación para garantizar el Plan Obligatorio de Salud en el Año 2009. Bogotá: Ministerio de la protección social; 2011.

14. Spitzer RL, Kroenke K, Williams JB. Validation and utility of a selfreport version of PRIME-MD: the PHQ primary care study. Primary Care Evaluation of Mental Disorders. Patient Health Questionnaire. JAMA. 1999;282(18):1737-44. doi:10.1001/ jama.282.18.1737.

15. Bellón Saameño JA, Delgado Sánchez A, Luna del Castillo JD, Lardelli Claret P. Validez y fiabilidad del cuestionario de función familiar APGAR. Aten Primaria.1996;18(6):289-96.

16. Bellón Saameño JA, Delgado Sánchez A, Luna del Castillo JD, Lardelli Claret P. Validez y fiabilidad del cuestionario de apoyo social funcional Duke-UNC-11. Aten Primaria. 1996.18(4):153-6.

17. Greenacre M. Correspondence analysis of raw data. Ecology. 2010;91(4):958-63.

18. Karlsson H, Joukamaa M, Lahti I, Lehtinen V, Kokki-Saarinen T. Frequent attender profiles: Different clinical subgroups among frequent attender patients in primary care. J Psychosom Res. 1997;42(2):157-66.

19. Gomes J, Machado A, Cavadas LF, Teixeira H, Pires P, Santos JA, et al. [The primary care frequent attender profile]. Acta Med Port. 2013;26(1):17-23. [Article in Portuguese]

20. Buja A, Toffanin R, Rigon S, Lion C, Sandonà $\mathrm{P}$, Carraro $\mathrm{D}$, et al. What determines frequent attendance at outof-hours primary care services?? Eur J 
Public Health. 2015;25(4):563-8. doi: 10.1093/eurpub/cku235.

21. Koskela TH, Ryynanen OP, Soini EJ. Risk factors for persistent frequent use of the primary health care services among frequent attenders: a Bayesian approach. ScandJPrim Health Care.2010;28(1):5561. doi: 10.3109/02813431003690596.

22. Hodgson P, Smith P, Brown T, Dowrick C. Stories from frequent attenders: a qualitative study in primary care. Ann Fam Med. 2005;3(4):318-23.

23. Mendoza-Sassi R, Béria JU. Utilización de los servicios de salud: una revisión sistemática sobre los factores relacionados. Cad Saude Publica. 2001;17(4):819-32.

24. Bergh H, Baigi A, Fridlund B, Marklund B. Life events, social support and sense of coherence among frequent attenders in primary health care. Public Health. 2006;120(3):229-36.
25. Guerra de Hoyos JA, de Anca Contreras IA. [Motives that condition use of the health services by over-users: study with focus groups]. Aten Primaria. 2007;39(7):349-54. [Article in Spanish]

26. Gili M, Luciano JV, Serrano MJ, Jiménez R, Bauza N, Roca M. Mental disorders among frequent attenders in primary care: a comparison with routine attenders. J Nerv Ment Dis. 2011;199(10):744-9. doi: 10.1097/ NMD.0b013e31822fcd4d.

27. Patel S, Kai J, Atha C, Avery A, Guo B, James M, et al. Clinical characteristics of persistent frequent attenders in primary care: case-control study. Fam Pract. 2015;32(6):624-30. doi: 10.1093/ fampra/cmv076.

28. Rincon-Hoyos HG, Rodriguez MR, Villa AM, Hernandez CA, Ramos ML. ¿Seria util la depuración de los trastornos mentales comunes en pacientes hiperfrecuentadores de servcios de salud en cuidado primario? Revista Colombiana de Psiquiatría. 2012;41(4):853-66. doi: 10.1016/ S0034-7450(14)60051-2.

29. Kendrick T, Pilling S. Common mental health disorders--identification and pathways to care: NICE clinical guideline. $\mathrm{Br} \mathrm{J}$ Gen Pract. 2012;62(594):47-9. doi: 10.3399/ bjgp12X616481.

Correspondencia: Mérida Rodriguez-López. Dirección: Sabino de Arana 1. Helios 3. Barcelona. España

Teléfono: +34666199445

Correo electrónico:merida.rdguez@gmail.com

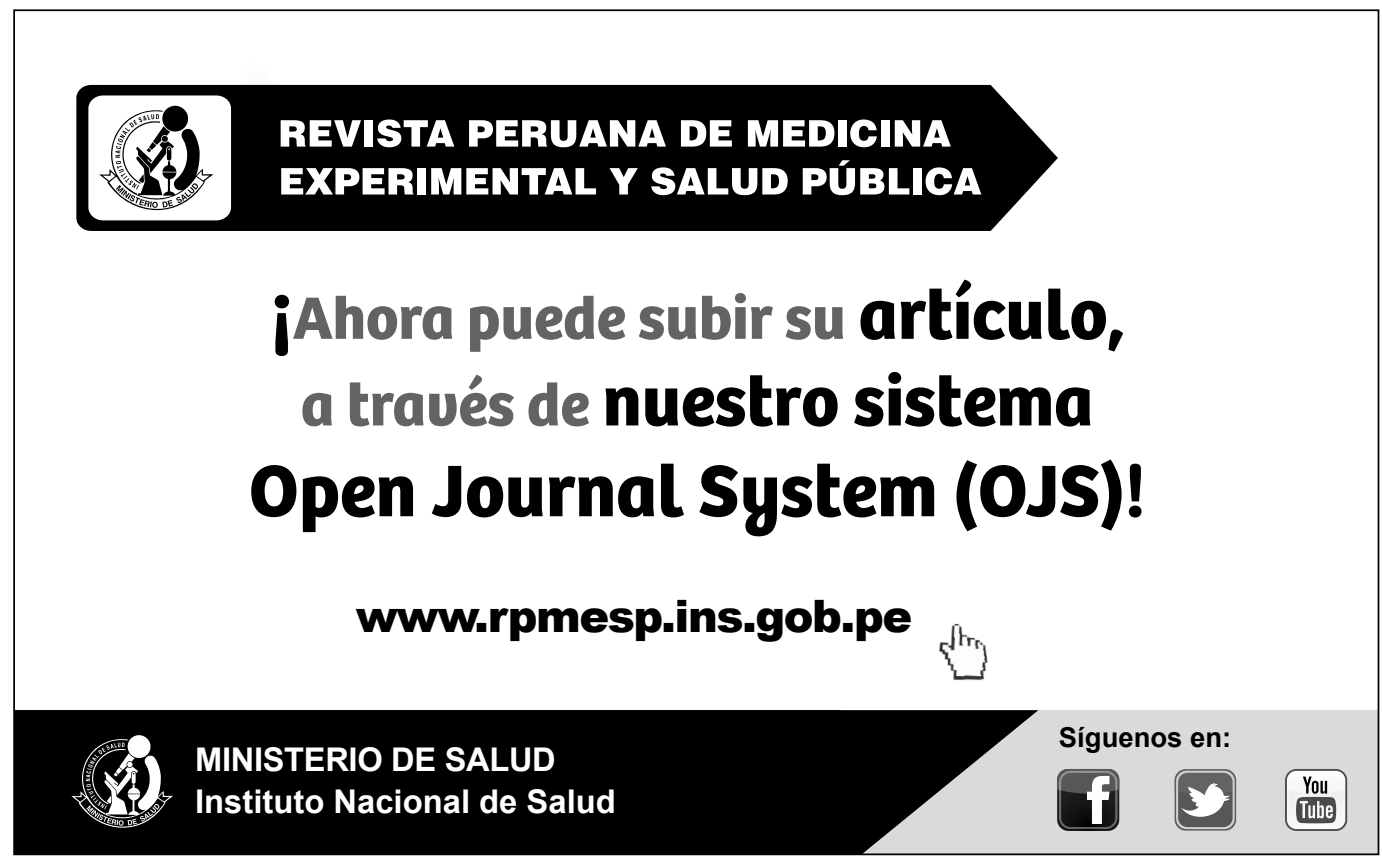

